\title{
Application of Fiber Bragg Grating Sensor for Strain Measurement at the Notch Tip under Cyclic Loading
}

\author{
Amirhossein Pahlevanpour, Bahareh Marzbanrad, Seyed Behzad Behravesh, Hamid Jahed \\ Mechanical and Mechatronics Engineering Department, University of Waterloo \\ Waterloo, Canada
}

\begin{abstract}
Notches are inevitable in many components and structures due to design limitations. In addition, they are the locations for stress concentration and are susceptible to fatigue failure. As a result, the cyclic stress/strain response at a notch is of key importance. Fiber Bragg Grating (FBG) sensors have been successfully utilized for mechanical and thermomechanical strain measurement in many cases; nevertheless, their capability of measuring strain at spots with intensive stress/strain has not yet been explored. In this research, FBG sensors are employed for strain measurement at the notch tip. A verification test was designed to substantiate the FBG measurements. The test involves a rectangular magnesium sheet with a center hole, subjected to uniaxial cyclic loading while the strain was measured at the notch tip using three different methods: strain gage, digital image correlation (DIC), and FBG. There were good agreements between the three measurements.
\end{abstract}

Keywords- Notch; strain measurement; FBG; DIC

\section{INTRODUCTION}

Fatigue is the dominant failure mode for components under cyclic loading, particularly in notched members [1]. To assess fatigue life, the cyclic stress/strain response at the notch tip is of key importance; however, strain measurement at this location has been challenging due to the high gradient of strain over a small area in the vicinity of the notch.

The common experimental approaches for notch stress/strain assessment are photoelasticity, thermoelasticity, brittle coatings, strain gages, and digital image correlation (DIC). Photoelasticity was initially devised by Brewster [2]. More than a century later, Coker and Filon developed this method as a tool for stress distribution assessment [3]. In this technique, a transparent material is utilized for fabricating a specimen with geometry similar to that of the notched member, and stress distribution is obtained by monitoring changes in the optical properties of the loaded transparent specimen via a polariscope. Photoelasticity can be adapted to both static and dynamic loading scenarios, even though doing so requires model preparation, and sometimes, tedious post-calculation [4].
The thermoelastic effect in metals was first recognized by Weber [5]. Belgen developed the first thermoelasticity application for contactless experimental stress analysis [6]. In thermoelasticity, local small temperature alterations of the loaded specimen indicate the stress distribution, allowing fullfield stress map to be made of the surface. In this method, the minimum detectable stress is restricted by the sensitivity of the employed apparatus; therefore, high precision requires costly experimental setup [7].

The brittle coating method begins with spraying a brittle coating on the surface of the notched member and letting it dry. The applied load will form crack patterns in the coating. The region and magnitude of stress concentrations can be indicated by comparing the crack patterns with the intact surface of the calibration coating. The coating's vulnerability to humidity and temperature, and the qualitative rather than quantitative results are two disadvantages of this method [8].

The strain gage technique is the most common experimental method for local strain measurement. The strain gage consists of a metallic foil pattern that acts as electrical resistance, supported by an insulating backing. It is attached to a prepared surface in the region of interest by a suitable adhesive. The dimensional change caused by the applied load results in electrical resistance alternation of the foil, which is used for strain measurement [9]. Initial calibration, nonlinearity, and pointwise measurement are among strain gage's main drawbacks [7].

Digital image correlation (DIC), is a non-contact optical technique for strain measurement, employs image tracking and registration techniques [10]. One of the first applications of DIC for deformation quantification was suggested by Peters and Ranson [11]. The DIC's accuracy has been widely substantiated through comparison with finite element and other verified methods [12]-[17]. Two DIC algorithms were used by Gonzáles and co-workers [10] for elastoplastic strain distribution measurement in shallow and deep notches. They verified their results with finite element method (FEM). Kotousov et al. [18] employed DIC for displacement- and strain-fields quantification near a sharp notch and compared the measurements with analytical and numerical solutions. 
The Fiber Bragg grating sensor is another advanced optical method for gauging external stimulants like strain. The Bragg gratings are written into the germanium-doped core of an optical fiber by periodic ultraviolet exposure leading to peculiar wavelengths reflection [19]. Any externally applied load and/or change in temperature increases or decreases the initial wavelength that is recorded by an interrogator during the experiment. The magnitude of this alternation can be converted into strain values by means of the mathematical expressions [19]. Even though the merit of FBG sensors for mechanical strain quantification has been verified extensively in the literature [20]-[24], its capability for intense notch tip strain measurement is still not well understood.

Because of the high resolution of the grating in the FBG sensor, it could be a proper candidate for mechanical strain measurement at spots with an intensive stress/strain gradient, e.g., at notches. To explore the applicability of the FBG, they are employed in this work for strain measurements at the notch tip. Variable amplitude cyclic loading was applied to the specimen, and the strain was measured utilizing three different measurement methods: strain gage, DIC, and FBG.

\section{MeAsurement AND TeSt PROCEDURES}

\section{A. Material and Experimental Setup}

The material under investigation was $4 \mathrm{~mm}$ thick AZ31BH24 hot-rolled magnesium sheet, provided by Magnesium Elektron of North America (MENA). Wrought magnesium has different yields in tension and compression [25] similar to the strength-differential effect seen in high strength steels [26]. The specimen was machined along the rolling direction with the dimensions shown in Fig. 1.

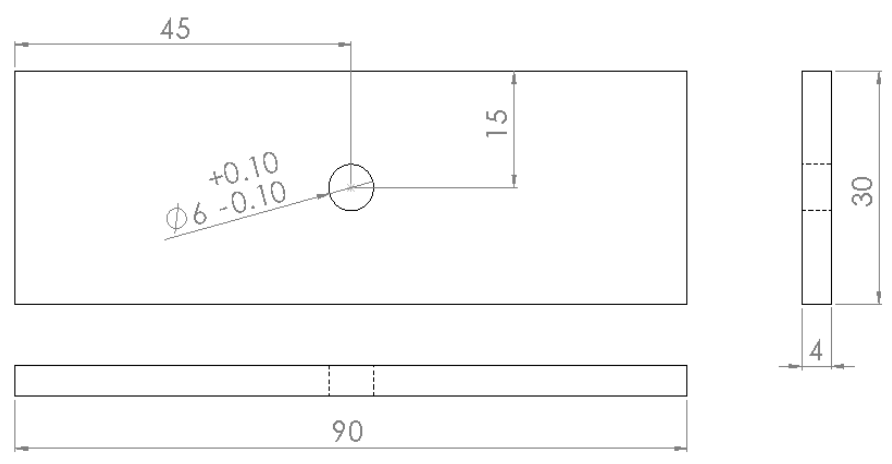

Figure 1. Notched specimen geometry (dimensions in millimeters)

Miniature strain gage C2A-06-015LW-120 from Micro Measurement Co. was selected for parallel strain measurement. The geometry of the strain gage is shown in Fig. 2. DAQDQ430 data-acquisition was employed, and one dummy gage was linked to the device to support quarter bridge gage configuration.

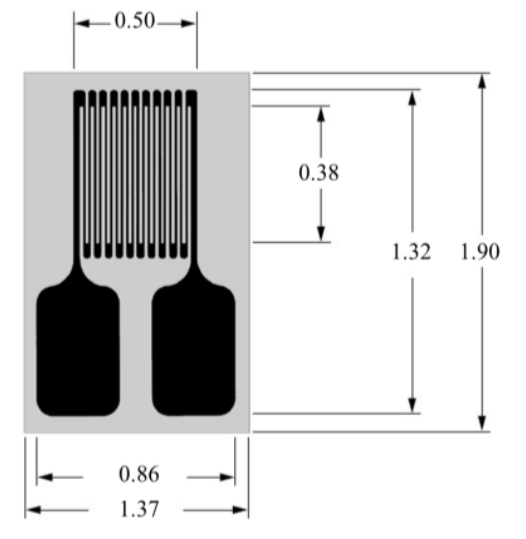

Figure 2. C2A-06-015LW-120 Strain Gage

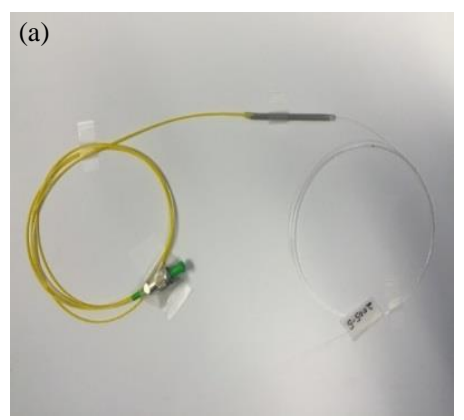

(b)

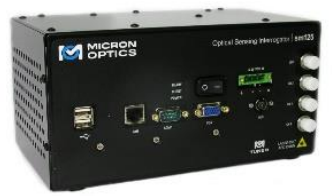

Figure 3. FBG equipment a) Corning SMF-28 with Bragg Grating b) Micron Optic SM125-200 Interrogator

Corning SMF-28 FBG with 5 mm effective length and 125 $\mu m$ dimeter were used in this research. The fiber optic sensor is illustrated in Fig. 3 (a). The initial wavelength was measured to be $1550 \mathrm{~nm}$. The grating modulation is Gaussian-apodized for this sensor, and the Bragg grating (1 mm length) had been written on a single mode of the optical fiber. The utilized optical interrogator is a Micron Optic SM125-200 with 5 pm resolution and an accuracy specification of $10 \mathrm{pm}$, as shown in Fig. 3 (b). It was connected to one side of the fiber optic cable by a FC/APC fiber connector.

For DIC strain measurement, the ARAMIS 3D System with two 5 megapixels CCD cameras, capable of $15 \mathrm{fps}$ readout at full resolution has been employed. This stereo system delivers precise 3D coordinates by using stochastic patterns or reference point markers.

Three different methods- DIC, strain gage, and FBG- were utilized for notch tip strain measurement. The schematic configuration indicating the devoted area to each technique is illustrated in Fig. 4 (a). To obtain comparable results from the different techniques, measurement was performed at the same distance from the notch tip. Due to the installation limitations enforced by the strain gage dimensions, the strain gage was attached to the specimen as close to the notch tip as was technically possible. Then, its location was used as a reference for the FBG sensor and DIC measurements. The FBG sensor was connected to the other side of the notch, as shown in Fig. 4 (b). For simultaneous strain measurement, LabVIEW was used. 


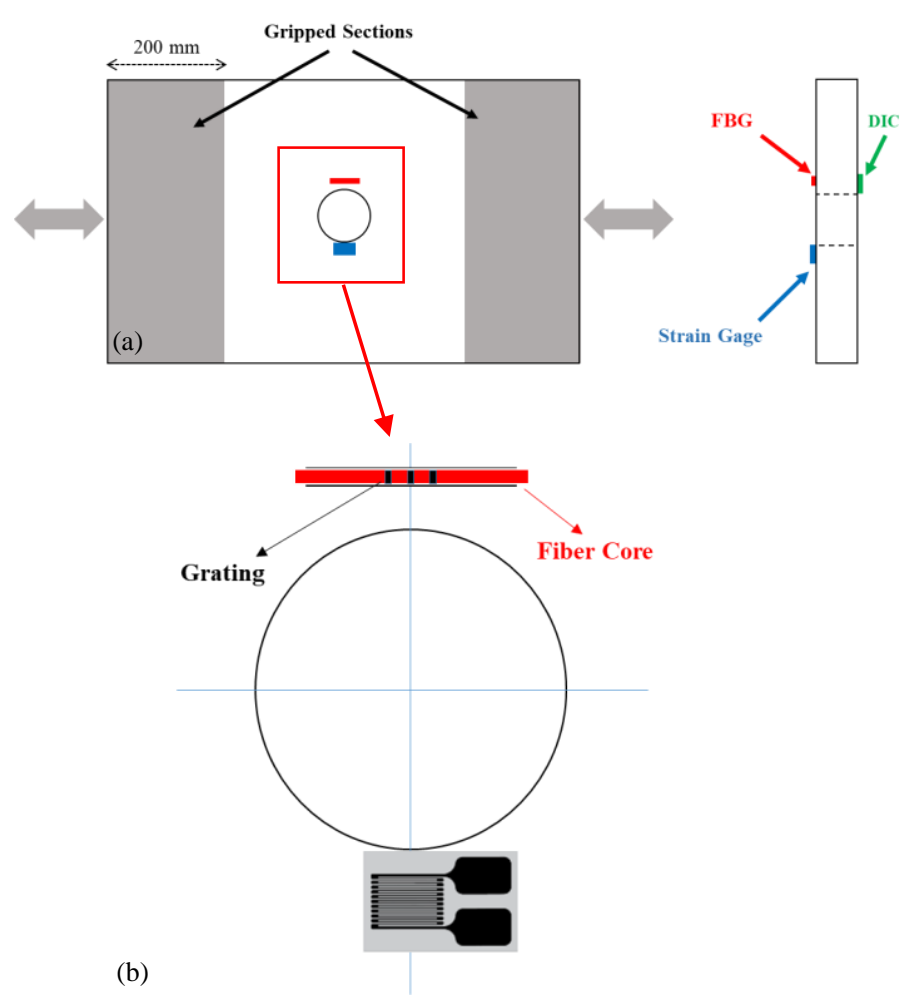

Figure 4. Schematic configurations of a) techniques used for notch tip strain measurement b) FBG and strain gage positions relative to the notch

\section{B. Test Procedures}

The specimen was loaded using the 8874 Instron servohydraulic test frame with $25 \mathrm{kN}$ load capacity and $100 \mathrm{~N} . \mathrm{m}$ torque capacity. The test was run in load-control mode, and a variable amplitude load (VAL) pattern was applied. The peak force was $13 \mathrm{KN}$ in accordance with the notch tip stress concentration to compel plastic deformation at the notch tip, while the far field remained elastic. Given the specimen geometry, shown in Fig.1, and the mode of loading, Fig. 4 (a), the stress concentration factor is equal to 2.5 [27]. The VAL loading scenario is depicted in Fig. 5.

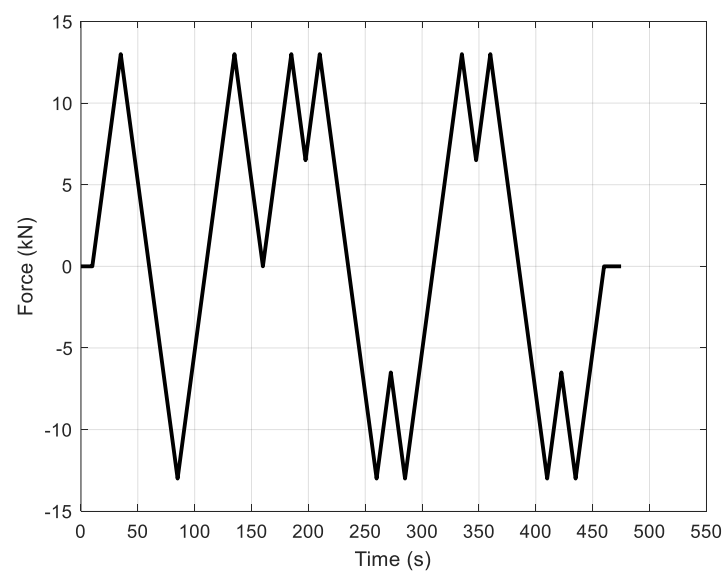

Figure 5. VAL scenario for FBG evaluation

\section{RESULTS AND DISCUSSION}

Fig. 6 shows the test setup. Measured Peak and valley strains are tabulated in Table. I, and the engineering strain as a function of time is plotted in Fig. 7. All three methods follow the VAL pattern closely, with DIC having more noise in the measurements. This issue can possibly be resolved by utilizing higher resolution cameras. Fig 8. manifests the strain distribution around the notch tip for the first peak of loading captured by DIC.
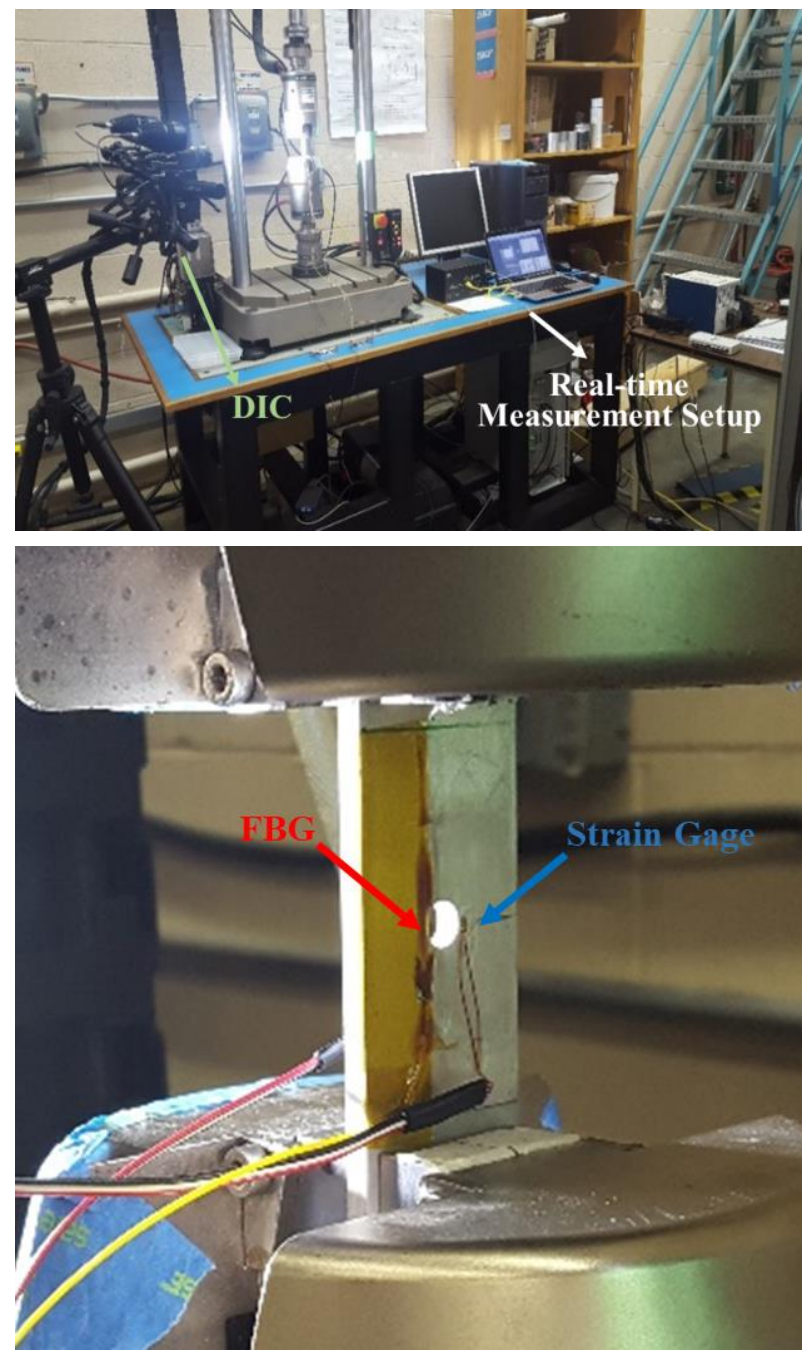

Figure 6. Final setup for simultaneous strain measurement

TABlE I. First Peak AND VALley Of StRain (\%) MEASURed By THREE DIFFERENT TECHNIQUES

\begin{tabular}{ccc} 
Techniques & Peak & Valley \\
\hline Strain Gage & 0.587 & -0.497 \\
\hline FBG & 0.601 & -0.575 \\
\hline DIC & 0.599 & -0.523
\end{tabular}




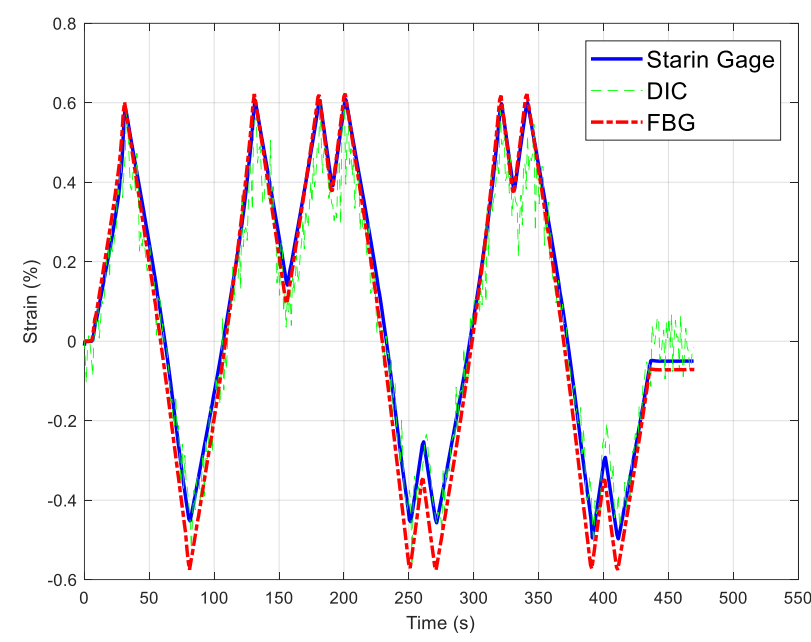

Figure 7. Notch strain measured by three experimental approaches

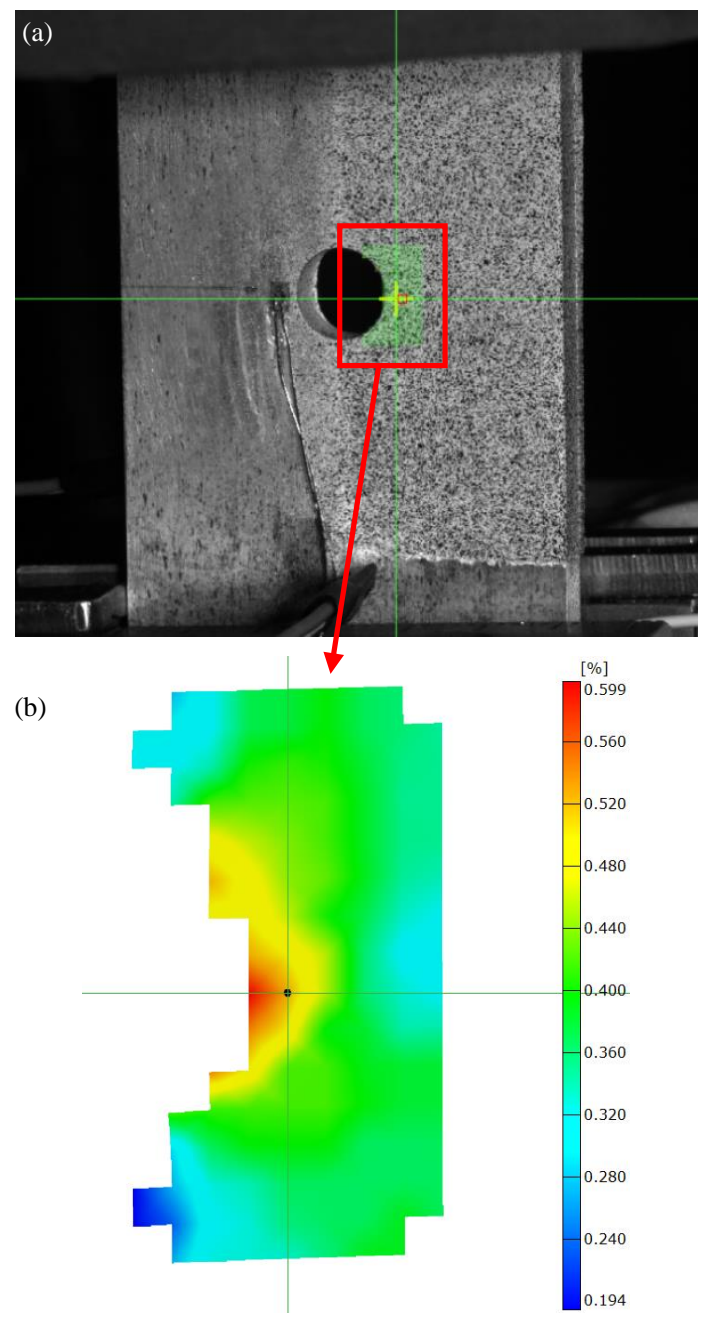

Figure 8. Peak strain distribution at the notch tip captured by DIC a) sample with speckle pattern b) strain map
The FBG sensor was capable of monitoring real-time strain during the experiment, whereas for DIC, the obtained images had to be analyzed after the test. In addition, the FBG pointwise resolution was higher compared to the strain gage. Overall, this study demonstrates the promising capability of FBG for fine strain measurement at the notch tip. Major sources for the slight difference in results are attributed to the following:

- Due to the very high strain gradient in the area near the notch root, even a few microns difference in the measurement position can affect the results significantly.

- All the implemented techniques average the strain over a specific area, e.g., the resistor area $\left(0.38 \times 0.5 \mathrm{~mm}^{2}\right)$ of the strain gage, although efforts have been made to keep it as small as possible.

\section{REFERENCES}

[1] R. I. Stephens, A. Fatemi, R. R. STEPHENS, and H. 0. FUCHS, Metal Fatigue in Engineering (second Edition), vol. 103, no. 4. 2001.

[2] D. Brewster, Experiments on the Depolarization of Light as Exhibited by Various Mineral, Animal, and Vegetable Bodies, with a Reference of the Phenomena to the General Principles of Polarization., vol. 2. 1815.

[3] E. G. Coker and L. Filon, Treatise on Photoelasticity. 1930.

[4] J. F. Doyle and J. W. Phillips, Manual on experimental stress analysis, vol. 5. 1989.

[5] W. Weber, "Über Die Spezifische Wärme Fester Körper Insbesondere Der Metalle," Ann. Phys. Chem, no. 96, pp. 177-213, 1830.

[6] M. H. Belgen, Infrared radiometric stress instrumentation application range study. 1968.

[7] W. N. J. Sharpe, Springer Handbook of Experimental Solid Mechanics. 2008.

[8] W. A. M. Brekelmans, H. W. Poort, and T. J. J. H. Slooff, "A new method to analyse the mechanical behaviour of skeletal parts," Acta Orthop., vol. 43, no. 5, pp. 301-317, 1972.

[9] M. Kutz, Handbook of measurement in science and engineering, vol. 3. 2016.

[10] H. Schreier, J. J. Orteu, and M. A. Sutton, Image correlation for shape, motion and deformation measurements: Basic concepts, theory and applications. 2009.

[11] W. H. Peters and W. F. Ranson, "Digital Imaging Techniques In Experimental Stress Analysis," Opt. Eng., vol. 21, no. 3, pp. 427-431, 1982

[12] B. W. Smith, X. Li, and W. Tong, "Error Assessment for Strain Mapping by Digital Image Correlation," Exp. Tech., vol. 22, no. 4, pp. $19-21,1998$

[13] W. Tong, "An Evaluation of Digital Image Correlation Criteria for Strain Mapping Applications," pp. 167-175, 2005.

[14] H. W. Schreier, J. R. Braasch, and M. A. Sutton, "Systematic errors in digital image correlation caused by intensity interpolation," Opt. Eng., vol. 39, no. November, p. 2915, 2000.

[15] Y. Q. Wang, M. A. Sutton, H. A. Bruck, and H. W. Schreier, "Quantitative error assessment in pattern matching: Effects of intensity pattern noise, interpolation, strain and image contrast on motion measurements," Strain, vol. 45, no. 2, pp. 160-178, 2009.

[16] B. Pan, A. Asundi, H. Xie, and J. Gao, "Digital image correlation using iterative least squares and pointwise least squares for displacement field and strain field measurements," Opt. Lasers Eng., vol. 47, no. 7-8, pp. 865-874, 2009.

[17] Z. Y. Wang, H. Q. Li, J. W. Tong, and J. T. Ruan, "Statistical analysis of the effect of intensity pattern noise on the displacement measurement precision of digital image correlation using self-correlated images," Exp. Mech., vol. 47, no. 5, pp. 701-707, 2007. 
[18] A. Kotousov, Z. He, and A. Fanciulli, "Application of digital image correlation technique for investigation of the displacement and strain fields within a sharp notch," Theor. Appl. Fract. Mech., vol. 79, pp. 5157, 2015.

[19] M. M. Werneck, R. C. Allil, and F. V. . Nazare, Fiber Bragg Gratings: Theory, Fabrication, and Applications. 2017.

[20] B. Marzbanrad, H. Jahed, and E. Toyserkani, "On the evolution of substrate's residual stress during cold spray process: A parametric study," Mater. Des., vol. 138, pp. 90-102, 2018.

[21] B. Marzbanrad, E. Toyserkani, and H. Jahed, "Cyclic hysteresis of AZ31B extrusion under load-control tests using embedded sensor technology," Fatigue Fract. Eng. Mater. Struct., vol. 40, no. 2, pp. 221232, Feb. 2017.

[22] B. Marzbanrad, H. Jahed, and E. Toyserkani, "On the sensitivity and repeatability of fiber Bragg grating sensors used in strain and material degradation measurement of magnesium alloys under cyclic loads," Int J. Adv. Manuf. Technol., vol. 86, no. 9-12, pp. 3453-3461, 2016.

[23] B. Marzbanrad, "Behaviour of Magnesium Alloy Under Load-Control Cyclic Testing," 2015.

[24] A. Feng, D. Chen, C. Li, and X. Gu, "Flat-cladding fiber Bragg grating sensors for large strain amplitude fatigue tests," Sensors, vol. 10, no. 8, pp. 7674-7680, Aug. 2010.

[25] S. B. Behravesh, H. Jahed, and S. Lambert, "Characterization of magnesium spot welds under tensile and cyclic loadings," Mater. Des., vol. 32, no. 10, pp. 4890-4900, 2011.

[26] J. Casey and H. Jahedmotlagh, "The strength-differential effect in plasticity," Int. J. Solids Struct., vol. 20, no. 4, pp. 377-393, 1984.

[27] W. C. Young and R. G. Budynas, Roark's Formulas for Stress and Strain, vol. 7, no. 7th Edition. 2002. 